\title{
Photofragmentation and electron detachment of aromatic phosphonate, sulfonate and phosphate oxyanions
}

\author{
Jennifer A. Noble ${ }^{1 *}$, Ernesto Marceca ${ }^{2}$, Claude Dedonder ${ }^{1}$, Isaure Carvin ${ }^{1}$, Eric Gloaguen ${ }^{3}$ \& Christophe \\ Jouvet $^{1}$
}

1 CNRS, Aix Marseille Univ., PIIM, Physique des Interactions loniques et Moléculaires, UMR 7345, 13397, Marseille, France. jennifer.noble@univ-amu.fr

2 INQUIMAE (CONICET - Universidad de Buenos Aires), DQIAQF (Facultad de Ciencias Exactas y Naturales, Universidad de Buenos Aires, Ciudad Universitaria), 3er piso, Pab. II, 1428 Buenos Aires, Argentina.

3 LIDYL, CEA, CNRS, Université Paris Saclay; CEA Saclay, Bât 522, 91191 Gif-sur-Yvette, France

\section{Abstract}

The photodetachment energy threshold, as well as vibrationally-resolved spectral signatures of the lower lying excited states and dipole bound states in model aromatic phosphonate, sulfonate and phosphate oxyanions have been investigated using a photofragmentation spectrometer equipped with a cold ion trap. The effect of the laser excitation was monitored by mass-selective detection of product ion fragments or, alternatively, measuring the yield of the complementary neutral radicals discriminated according to their kinetic energy. The anions phenylphosphate, phenylsulfonate and p-toluenesulfonate evidenced the expected behaviour, characterised by the predominance of ionic fragmentation processes, at low energies, rapidly evolving to a scenario controlled by the electron photodetachment channel at higher energies. Surprisingly for such a similar system, the phenylphosphonate anion does not have any ionic fragmentation channels and only exhibits the presence of dipole bound states.

\section{Introduction}

The photophysical properties of isolated aromatic carboxylates $\left(\mathrm{Ar}-\mathrm{CO}_{2}{ }^{-}\right.$, with $\mathrm{Ar}$ representing the aryl residue) have recently been explored in the UV region of the spectrum. ${ }^{1,2}$ In such systems, photoexcitation has been shown to lead to a competition between electron detachment followed by decarboxylation, and dissociative ionic channels producing neutral and anionic fragments. However, there is no information available in the literature about the gas phase spectroscopic behaviour of analogous oxyanions containing phosphorus or sulfur atoms such as, for example, aryl phosphonates $\left(\mathrm{Ar}_{-} \mathrm{PO}_{3} \mathrm{H}^{-}\right)$and sulfonates $\left(\mathrm{Ar}-\mathrm{SO}_{3}{ }^{-}\right)$. Experimental access to the absorption spectrum and the electron detachment energy of these anions would contribute to the understanding of how the groups $-\mathrm{CO}_{2}{ }^{-},-\mathrm{PO}_{3} \mathrm{H}^{-}$and $-\mathrm{SO}_{3}{ }^{-}$perturb lower lying electronic states, especially taking into account the fact that their characterisation has thus far remained elusive even to high level electronic structure calculations. ${ }^{3}$

Negative ion electrospray mass spectrometry studies have demonstrated that alkyl phosphonates (for example, aminomethyl phosphonate $\mathrm{H}_{2} \mathrm{~N}-\mathrm{CH}_{2}-\mathrm{PO}_{3} \mathrm{H}^{-}$) lose the $\mathrm{PO}_{3}{ }^{-}(\mathrm{m} / z=79$ ) group, ${ }^{4}$ with a suggested mechanism for the $\mathrm{P}-\mathrm{O}$ bond cleavage involving a four-membered $\mathrm{CPOH}$ transition state, which evolves with a concomitant hydrogen migration and formation of the molecule $\mathrm{H}_{2} \mathrm{~N}-\mathrm{CH}_{3}$. No information is available to support whether this fragmentation channel will also occur in aryl phosphonates. On the other hand, it is known that the collisionally activated dissociation of the $\mathrm{S}-\mathrm{O}$ bond in aryl sulfonate anions is majorly homolytic, producing the corresponding aryl radical and the $\mathrm{SO}_{3}{ }^{-}$radical anion. ${ }^{5,6}$

A second category of aryl oxyanions is attained by phosphorylation or sulfation of aromatic alcohols, which yields aryl phosphate $\left(\mathrm{Ar}-\mathrm{O}-\mathrm{PO}_{3} \mathrm{H}^{-}\right)$, or aryl sulphate $\left(\mathrm{Ar}-\mathrm{O}-\mathrm{SO}_{3}{ }^{-}\right)$, ester anions. These 
anions are particularly widespread and important in biological systems, where enzyme-catalysed phosphorylation and sulfation reactions of carbohydrates, proteins, and glycoproteins participate in crucial processes such as cell signalling, hormone regulation and molecular recognition. In particular, protein phosphorylation and, to a lesser degree, sulfation are abundant post-translational modifications that proceed along kinase, or sulfotransferase, enzymatic mechanisms. These reactions can occur on the side chain - $\mathrm{OH}$ groups of serine and threonine as well as on the aromatic - $\mathrm{OH}$ group of tyrosine, and it is through the regulation of these processes that the diversity of the biological functions of these molecules drastically increases. Particularly, phosphorylation of tyrosine residues can be driven by specific kinases that govern many important cell mechanisms.

The role of the phosphate group on the absorption spectrum of tyrosine was recently investigated ${ }^{7,8}$. For the protonated peptide - for which the phosphate group is expected to be neutral, adopting the structure of the $-\mathrm{O}-\mathrm{PO}(\mathrm{OH})_{2}$ conjugated acid- it was observed that the absorption of tyrosine shifts from $\approx 35000 \mathrm{~cm}^{-1}$ to $37000 \mathrm{~cm}^{-1}$ for the phosphorylated tyrosine. However, the effect of the phosphate's charge state on the absorption of the phenol chromophore of tyrosine still remains unknown. Owing to the importance of this group and its large number of possible charge states, we would expect to find many literature references to these systems, such as the measurement of the Adiabatic Detachment Energy (ADE) or the different charge states, but we found only the measurement by photoelectron spectroscopy ${ }^{9}$ of the $A D E$ for $\mathrm{H}_{2} \mathrm{PO}_{4}^{-}$at $4.57 \mathrm{eV}$ and $\mathrm{HPO}_{4}{ }^{2-}$. For comparison we have studied similar aromatics with a sulfate group to see how the absorption of the anion changes with functional group. The $\mathrm{HSO}_{4}^{-}$group itself has an $\mathrm{ADE}=4.75 \mathrm{eV}^{10}$.

Finally, the PO group has also been recently observed ${ }^{11}$ in the interstellar medium and, in addition to the discovery of prebiotic atoms $(\mathrm{C}, \mathrm{H}, \mathrm{N}, \mathrm{O}$, etc) and molecules (glycine, etc), this considerably reinforces the panspermia paradigm ${ }^{12}$ !

The photoexcitation of an anion is quite complex since many competitive processes can occur. At low energy, there is only access to a limited number of valence electronic excited states that lie below the adiabatic detachment energy (ADE). These states can either fluoresce ${ }^{13}$ or dissociate producing an anionic fragment and a neutral particle. If dissociation takes place, the kinetic energy profile acquired by the fragments can be accounted for in the arrival time profile of neutral particles measured at a detector sufficiently far away (e.g. in a time of flight (ToF) mass spectrometer). Above the ADE, the anions have a different type of electronic excited states that consist of autodetaching resonances, which remain coupled to the electron detachment continuum threshold. An interesting discussion about the nature of such resonances can be found elsewhere ${ }^{14-}$ ${ }^{16}$. For energies greater than the $A D E$, the newly opened electron detachment channel competes with anion fragmentation, which can still occur. As the photon energy increases, electron photodetachment becomes ever faster and it soon becomes the dominant process. Moreover, once the electron is detached from the anion, the resulting neutral radical species may potentially dissociate giving rise to two neutral fragments ${ }^{1}$. In addition to valence excited states and autodetaching resonances, molecular anions can be excited to dipole bound states (DBS), which resemble the Rydberg states in neutral molecules. Dipole bound states are stable and well characterised when the dipole of the radical is larger than $4 D^{17-23}$. They will vibrationally auto-ionise when the vibrational states of the DBS are higher than the $A D E$, leading to a neutral radical.

In this work, we measured photofragmentation and photodetachment spectra of phenylphosphonate, phenylsulfonate, $\mathrm{p}$-toluenesulfonate and phenylphosphate monoanions, and interpreted the data with assistance of electronic structure calculations. We used a laser spectrometer equipped with a cold trap that has the capability to detect both product ions or neutral particles issued from photodetachment and/or photofragmentation. The experimental data allows us to study the lower lying anionic electronic states, measure electron adiabatic detachment energies, and characterise the presence of DBSs. A particular focus was the assignment of the spectral features to each of the competing deactivation processes of the excited anions, i.e. electron detachment (and, potentially, subsequent neutral fragmentation) vs. ionic fragmentation. 


\section{Experimental methods}

The experimental setup used to measure cold ion photofragmentation (PF) and photodetachment (PD) spectra was described in previous publications ${ }^{24-26}$. The apparatus was recently modified to detect negative ions and the neutral particles originating from both the electron detachment process and following fragmentation ${ }^{1,2}$. The oxyanions are produced in an electrospray ionisation (ESI) source by deprotonation of phenylphosphonic, phenylphosphoric, phenylsulfonic, and p-toluenesulfonic acids. In each case, a $10^{-4} \mathrm{M}$ solution of the corresponding acid in a $5: 1$ mixture of methanol:water is injected into the ESI source. The negative deprotonated ions produced in the ESI source are then guided into the cryogenic ion trap, just after a helium gas pulse. The ions are stored in the trap for a few tens of milliseconds, the time necessary to cool them down to ca. 30 $\mathrm{K}$ by collisions, then to ensure the reduction of the pressure in the trap via the pumping system.

The ions are then extracted and accelerated to $3.0 \mathrm{kV}$. The voltage of the extracting electrode of the trap and that of the accelerating grid are adjusted to fulfil the Wiley McLaren focusing conditions $^{27}$. Once they pass through the accelerating grid, the ions enter a Gauss tube set at the accelerating grid potential and, once they are inside, the tube is switched to ground. By doing that, the ions travel the length of the field free region of the ToF mass spectrometer with the kinetic energy acquired at the acceleration stage while remaining referenced to the ground potential. After $1 \mathrm{~m}$ of flight, the particles can be post accelerated/decelerated and are finally detected with a MCPbased detector ${ }^{28}$.

The laser used for photodissociation or photodetachment of the ions is a tuneable OPO laser (EKSPLA, $10 \mathrm{~Hz}$ repetition rate, $10 \mathrm{~ns}$ pulse width, and a spectral resolution of $\sim 10 \mathrm{~cm}^{-1}$ ). The laser can interact with the ions in two different parts of the setup, depending on the type of experiment that is being carried out:

A) Anion yield vs. excitation energy spectrum (ion detection). In this case, the ion-laser interaction occurs while the ions are inside the cold trap. In this configuration, the precursor and product ions generated in the dissociation process are extracted immediately after the laser shot, they are mass analysed in the TOF spectrometer, and finally quantified. Using this procedure, the PF spectrum can be recorded by following an identified photoproduct's yield.

B) Neutral particle yield vs. excitation energy spectrum (neutral detection). In this experiment, the ions are excited inside the Gauss tube. Since, at that point, precursor anions possess a high kinetic energy, the intact radicals that result from electron PD can be counted at the MCP detector, together with any neutral fragments that are also formed after PD. The yield of intact and fragment neutrals can largely be distinguished by considering their distinct arrival time at the detector. On the one hand, unfragmented radicals arrive at the detector at the arrival time of the precursor anion, and the signal is temporally as narrow as the original anion signal. In contrast, neutral fragments travel with an excess kinetic energy (positive or negative) released in the dissociative process, so that they hit the detector at the arrival time of the precursor anion, but the peak exhibits a significant signal broadening. When operating in neutral detection mode, the ions are deflected away from the detector, and only the yield of neutral particles is quantified. By applying this method, the PD process becomes experimentally accessible and the ADE can be determined by observing the onset of the narrow central peak corresponding to the arrival of neutral radicals at the detector (see the examples in the $\mathrm{SI}$ and in our recent publications ${ }^{1,2}$ ).

In both experiments, the laser interacts only once with the ion cloud, the signal is usually averaged over 8 shots at each wavelength, the spectrum is normally recorded ten times (less, if the signal is very good and the source very stable), and then averaged. In the following figures no smoothing algorithm was employed, and the observed noise is due either to the statistical uncertainty and/or to the instability of the source. Ion/neutral yield scans are obtained by measuring the signal within a temporal window corresponding to the anion or neutral radical (narrow peak) and/or the neutral 
fragments (broad peak) at each excitation wavelength; the temporal profiles are measured at a single wavelength then symmetrized, as described in our recent publications. ${ }^{1,2}$

\section{Experimental Results}

The presentation of measured PF and PD spectra is organized into two sections, according to the molecular anion type (chemical family), i.e. phosphonate/sulfonate anions, and phosphate ester anions. The figures and inserts may include up to three types of data: i) anion yield spectrum (ion detection), in which the intensity represents the yield of an identified negatively charged photofragment, ii) neutral particle yield spectrum (neutral detection), in which the yield of neutral fragments arising from anion/radical dissociation is separated from the yield of the intact radical issued from electron photodetachment, and iii) temporal profile of the neutral peak at the detector, which allows us to distinguish between PF and PD processes.

\subsection{Aromatic phosphonates and sulfonates}

\section{Phenylphosphonate anion $\left(\mathrm{Ph}-\mathrm{PO}_{3} \mathrm{H}^{-}\right)$}

Under our electrospray conditions, the only fragment observed in the trap is $\mathrm{PO}_{3}{ }^{-}$, which has a very weak signal of around $0.3 \%$ that of the parent ion (ion yield spectrum shown in Fig. SI-1). Although weak, this fragmentation resembles that observed for alkyl phosphonates, ${ }^{4}$ involving probably a four-membered $\mathrm{CPOH}$ transition state, and where a hydrogen atom is transferred from the phosphate group to the aromatic ring leading to the formation of benzene.

Most of the experiments performed on this anion are summarised in Fig. 1. The limited fragmentation of $\mathrm{Ph}_{-} \mathrm{PO}_{3} \mathrm{H}^{-}$is evidenced not only by a poor yield in the ion detection spectrum, but also by the shape of the temporal profile of neutrals, recorded at $266 \mathrm{~nm}$ and higher energies (insert at the right of Fig. 1). It can be observed that the profile appears essentially as a narrow peak, indicating that the fragmentation channel is almost closed. 


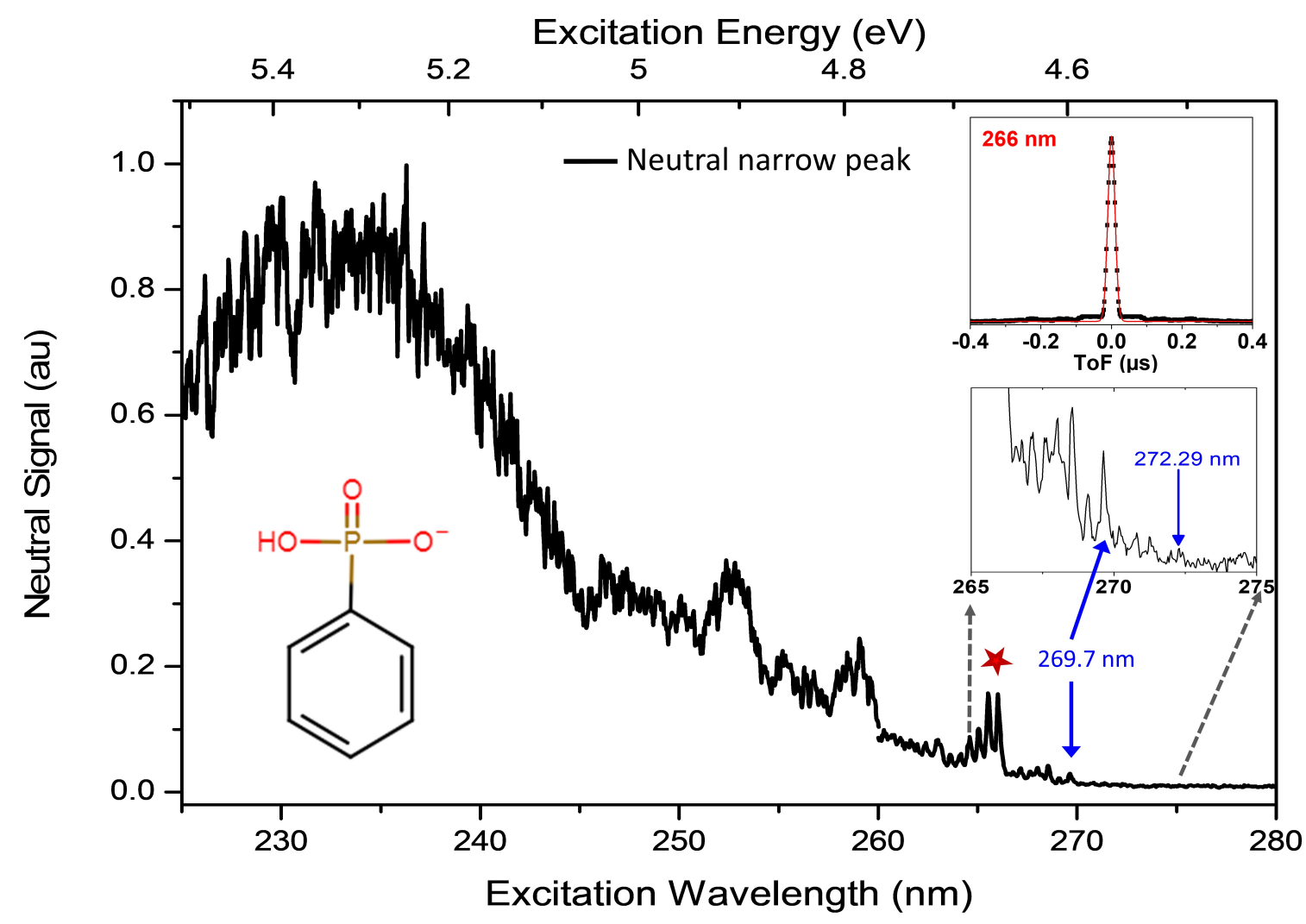

Figure 1. Neutral particle yield spectrum of the phenylphosphonate anion (main black trace). The vibrational structure observed in the 260-270 nm region is not present in the ion detection spectrum (Fig. SI-1). The Insert in the lower right shows a magnification of the low energy region. The insert in the upper right shows the temporal profile of neutrals arriving at the detector, upon excitation of the first intense absorption band at 266 $\mathrm{nm}$ (marked in the spectrum with a star; data are presented in black and the red curve is a Gaussian fit).

The neutral particle yield spectrum in Fig. 1 shows the UV absorption of the anion, with a first clear band located at $269.7 \mathrm{~nm}(4.59 \mathrm{eV})$, although weaker bands can be identified at lower energies, starting at $272.29 \mathrm{~nm}(4.56 \mathrm{eV})$. In the range between 270 and $266 \mathrm{~nm}$ a complicated low frequency vibrational structure is observed, with a simple vibrational progression of $70 \mathrm{~cm}^{-1}$ distinguished near $266 \mathrm{~nm}$. These bands are assigned to the vibrations of a DBS, as will be discussed later in further detail. At higher energy, below $266 \mathrm{~nm}(>4.66 \mathrm{eV}$ ), several broad absorption bands are observed, with still no sign of fragmentation. No detachment threshold with the characteristic step shape is visible in the spectrum, and thus it is not possible to determine the value of the ADE directly. However, as will be presented in section 5, an estimation of the ADE can still be obtained from the analysis of the DBS spectrum.

\section{Phenylsulfonate anion $\left(\mathrm{Ph}_{-} \mathrm{SO}_{3}^{-}\right)$}

In accordance with previous collisionally-activated dissociation experiments, PF of the Ph$\mathrm{SO}_{3}{ }^{-}$anion proceeds via $\mathrm{SO}_{3}{ }^{-}$loss. ${ }^{6,29}$ The spectrum obtained by plotting the $\mathrm{SO}_{3}{ }^{-}$ion yield vs. the excitation energy is identical to the one recording the intensity of neutrals corresponding to the temporal profile edge, i.e. where the signal is a measure of neutrals originated in a dissociation process (having an excess of kinetic energy). Because of that, we will focus on the neutral detection spectrum, plotted in Fig. 2. 


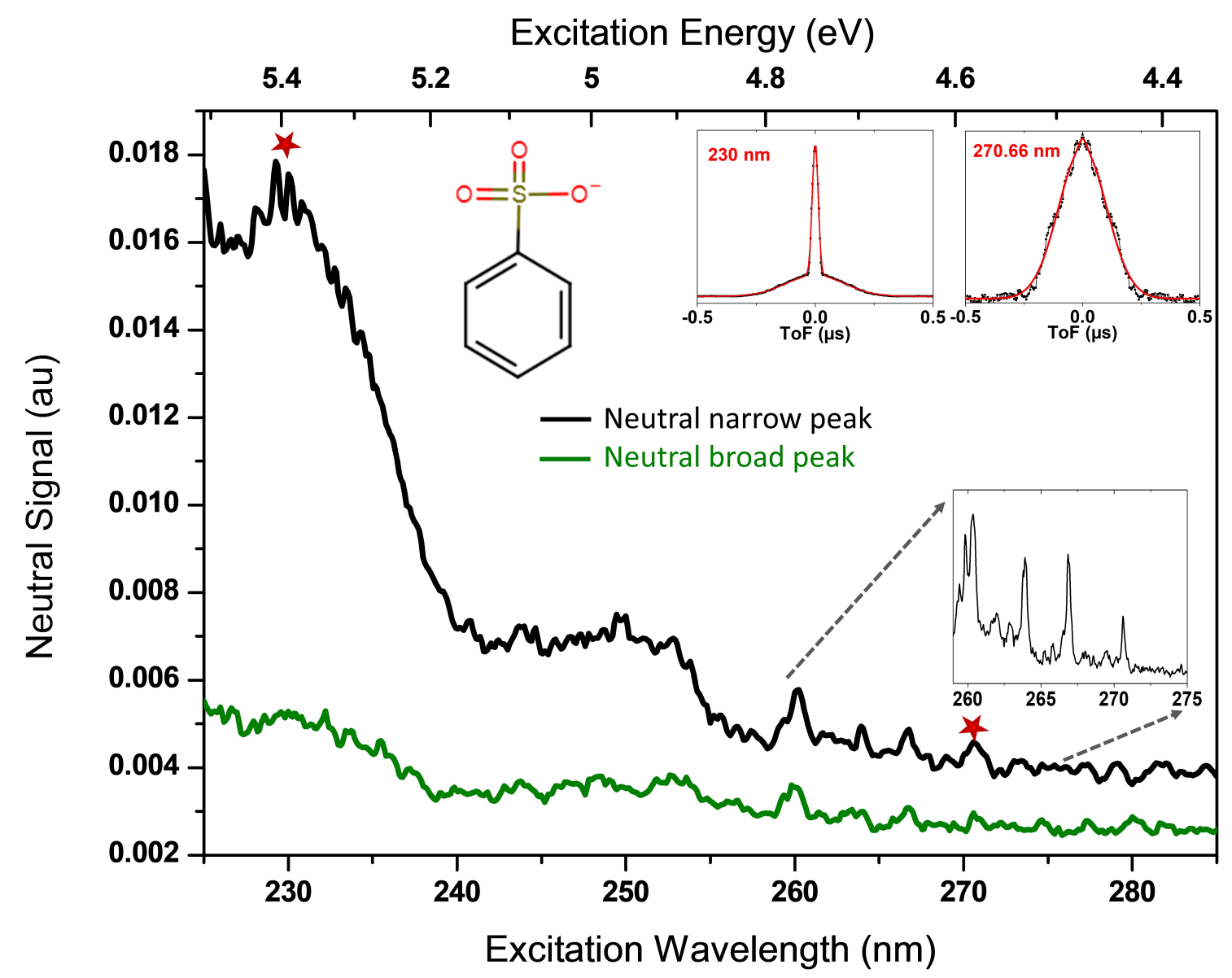

Figure 2. Neutral particle yield spectrum of the phenylsulfonate anion. Neutrals mostly originate in: i) PD process (black), i.e. sampled at the central peak of the temporal profile, and ii) PF process (green), i.e. sampled at the edge of the temporal profile. The insert in the lower right represents the beginning of the spectrum, at higher resolution. Experimental ADE threshold observed at $253.5 \pm 0.05 \mathrm{~nm}$. The insets in the upper right show the temporal profile of neutrals arriving at the detector upon excitation at $230 \mathrm{~nm}$ and on the first band at 270.66 $\mathrm{nm}$ (experimental data are in black, and in red the fitted profile).

In contrast to phenylphosphonate, a broad temporal profile is observed for neutrals released from the phenylsulfonate anion, indicating that these neutrals are produced by fragmentation. A detailed observation of the profile (see e.g. that obtained upon excitation at $230 \mathrm{~nm}$ in the upper right of Fig. 2) reveals that it has two components with different temporal widths (i.e. kinetic energy dispersion): a broad part due to neutral fragments, and a narrow central part attributed to the stable radical remaining after electron $\mathrm{PD}$. The relative contribution of these two processes to the profile is found to be dependent on the excitation energy. For example, at excitation energies of about 270 $\mathrm{nm}$, a broad temporal dispersion is found and fully explained by solely PF channels while, at higher excitation energies, electron PD tends to become the dominant process.

The action spectra sampled on the central part (black trace) and on the edge (green trace) of the temporal profile are very similar from $280 \mathrm{~nm}$ up to $253.5 \mathrm{~nm}$, because in this spectral region the electron detachment channel is closed. At higher energies, however, this situation changes and the contribution of the PD channel (black trace) increases, with a clear onset at $253.5 \pm 0.05 \mathrm{~nm}(4.89 \mathrm{eV})$ (see Fig SI-3). This value results a very precise measure of the ADE for this molecular anion. Other important characteristics of the spectrum are represented in the insert in the lower right of Fig. 2 , acquired at higher resolution. The first observed band, located at $270.66 \mathrm{~nm}\left(36949 \mathrm{~cm}^{-1}\right)$, is associated with two vibrations at $515 \mathrm{~cm}^{-1}$ and $939 \mathrm{~cm}^{-1}$, which are assigned to breathing modes of 
the phenyl ring $\left(v_{10}=575 \mathrm{~cm}^{-1}\right.$ and $v_{20}=1008 \mathrm{~cm}^{-1}$ in the ground state). These vibrations have very similar values to those characteristic of the aromatic molecules benzene or toluene.

\section{p-toluenesulfonate anion $\left(\mathrm{CH}_{3}-\mathrm{Ph}-\mathrm{SO}_{3}{ }^{-}\right)$}

This molecular anion is structurally similar to phenylsulfonate and hence the photophysical behaviour is expected to be analogous. In fact, $\mathrm{PF}$ of $\mathrm{CH}_{3}-\mathrm{Ph}_{-} \mathrm{SO}_{3}{ }^{-}$is again characterised by a $\mathrm{SO}_{3}{ }^{-}$loss, and the spectrum measured on this ion channel coincides with the one recorded by sampling neutral fragments at the edge of the temporal profile. As we did before, we will focus on the fragmented neutral particle detection spectrum, given in Fig. 3.

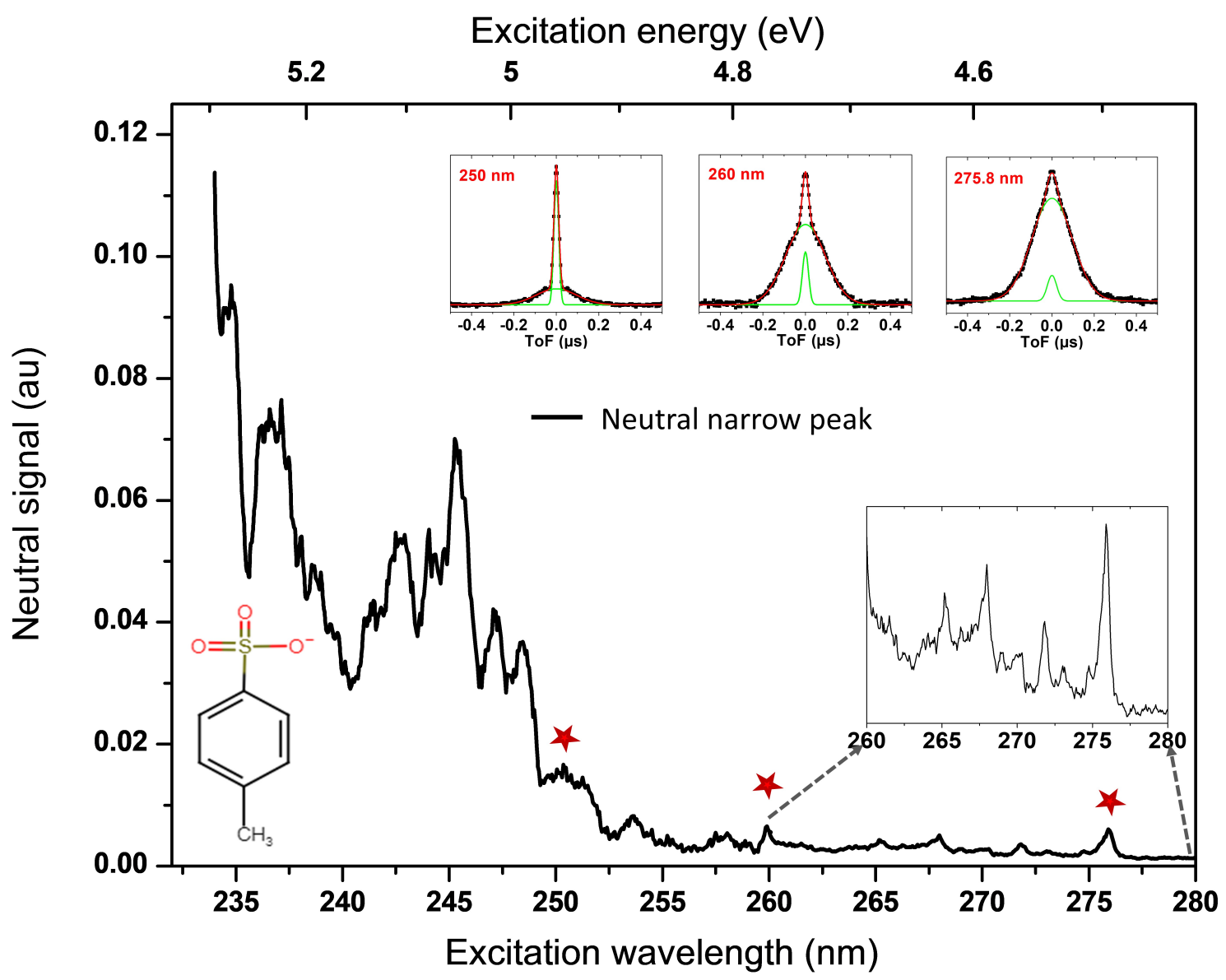

Figure 3. Neutral particle yield spectrum of the p-toluenesulfonate anion. Neutrals essentially originate from PD (sampled at the central peak of the temporal profile), but there is also a minor contribution of the neutral broad peak at low energies as presented in Fig SI-4. The insert at the lower right represents the beginning of the spectrum, at higher resolution. The experimental ADE threshold is observed at $261.5 \pm 0.05 \mathrm{~nm}$. The inserts in the upper right show the temporal profile of neutrals arriving at the detector upon excitation at $250 \mathrm{~nm}, 260 \mathrm{~nm}$ and on the first band at $275.8 \mathrm{~nm}$ (experimental data are in black, and in red and green the fitted profile and its components, respectively).

As in the case of phenylsulfonate, the broad component of the temporal profile of neutral fragments is dominant at low energy (e.g. upon excitation at $275.80 \mathrm{~nm}$ ) and becomes relatively smaller at higher energies (e.g. at $250 \mathrm{~nm}$ ). For this molecular anion, it was also possible to determine a value of $261.5 \pm 0.05 \mathrm{~nm}(4.74 \pm 0.01 \mathrm{eV})$ for the ADE, from a sudden increase in the signal recorded by sampling unfragmented neutrals (central part of the profile). 


\subsection{Aromatic phosphate}

\section{Phenylphosphate ester anion ( $\mathrm{Ph}-\mathrm{O}-\mathrm{PO}_{3} \mathrm{H}^{-}$)}

Phosphate anions behave differently than phosphonates, because the O-P bond of the phosphoester is more susceptible to break upon excitation than phosphonate's C-P bond. Although the major dissociation channel of phenylphosphonate and phenylphosphate consists of releasing the same ion fragment, $\mathrm{PO}_{3}^{-}$, the yield is much larger for the latter. It was observed that the $\mathrm{PO}_{3}{ }^{-}$ion yield spectrum has an intensity of about $20 \%$ that of the precursor ion, by comparing the intensity of the main peaks. The fragmentation mechanism involving the release of $\mathrm{PO}_{3}^{-}$ions probably evolves via a four-membered transition state, by transferring a hydrogen atom from the phosphate group to the phenoxy moiety, with the formation of phenol. As shown in Fig. 4 (lower panel), the $\mathrm{PO}_{3}{ }^{-}$ fragment signal exhibits two clear vibrational progressions of narrow bands; one of them starts in a weak band at $290.8 \mathrm{~nm}$, and the other in a much more intense band at $280.68 \mathrm{~nm}$. In accordance with the behaviour of sulfonates, the ionic fragmentation signal vanishes when the photon energy increases, i.e. below $260 \mathrm{~nm}$, once the competing electron PD channel is fully open.

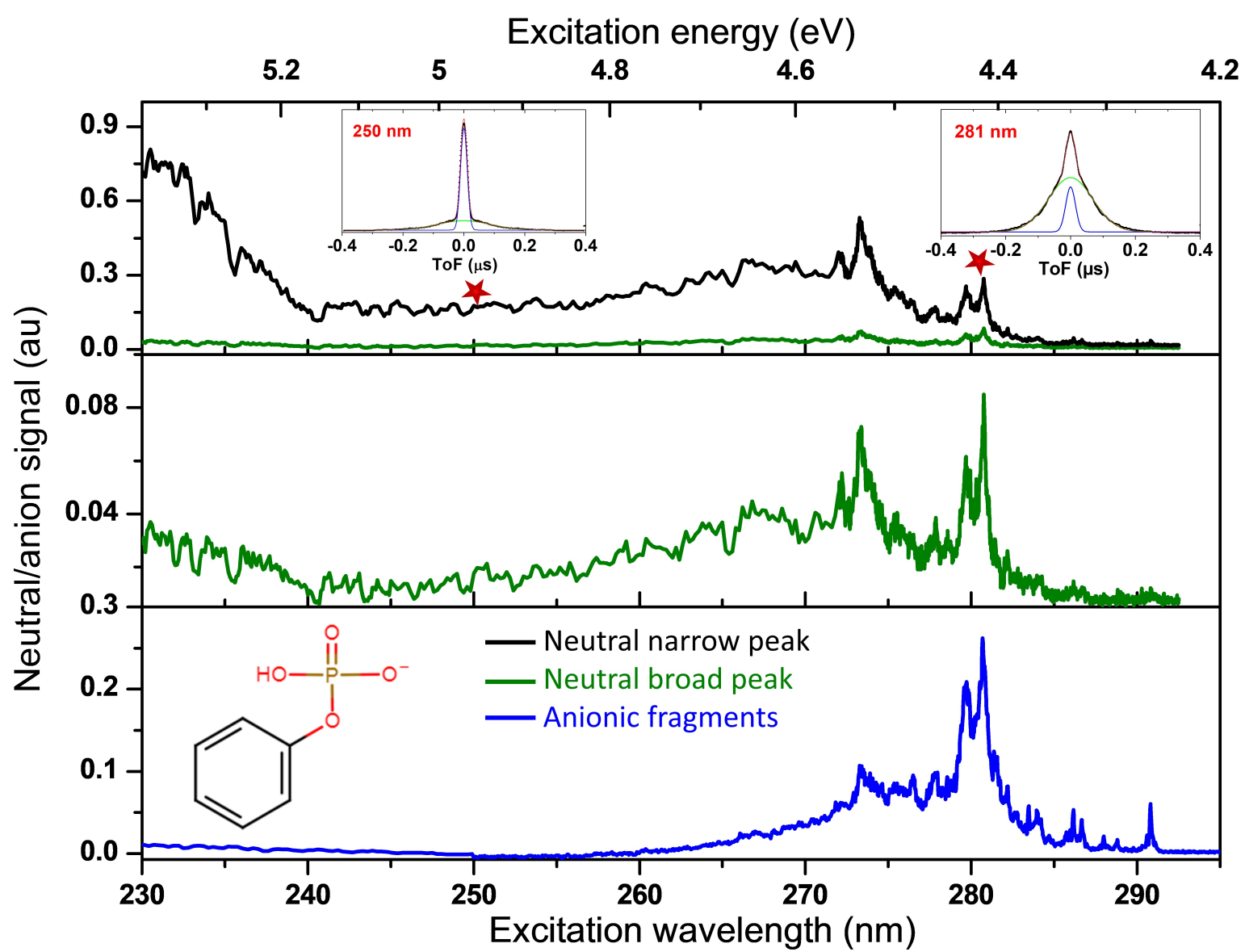

Figure 4. $\mathrm{PO}_{3}{ }^{-}$ion yield (lower panel) and neutral particle yield (middle and upper panels) spectra of phenylphosphate anion. Neutrals mostly originate in: i) PD process (black), i.e. sampled at the central peak of the temporal profile, and ii) PF process (green), i.e. sampled at the edge of the temporal profile. Insets in the upper panel show the temporal profile of neutrals arriving at the detector upon excitation at $250 \mathrm{~nm}$ and at 281 $\mathrm{nm}$ (experimental data are in black, and in colour the fitted profile).

The fragmentation of phenylphosphate is also evidenced in the temporal profiles of neutrals shown as insets in the upper panel of Fig. 4, in which an active dissociative channel (broad contribution to the peak) is observed for an excitation energy corresponding to $281 \mathrm{~nm}$. 
The action spectrum sampled on the central peak of the temporal profile of neutrals is shown in the upper panel of Fig. 4 (black trace). At low energy, the spectrum is similar to the $\mathrm{PO}_{3}{ }^{-}$ion yield spectrum (lower panel), but at high energy $(<240 \mathrm{~nm})$ a spectrally broad and intense band appears, which is not seen in the ion yield spectrum. If now the action spectra of neutrals is sampled on the edge of the temporal profile (green trace, middle and upper panels), the appearance changes drastically and there is only very low fragmentation in the UV, exactly as in the $\mathrm{PO}_{3}{ }^{-}$ion yield spectrum.

As we discussed earlier, the experimental access to the ADE of phenylphosphate relies on determining at which excitation energy the central peak contribution becomes visible in the temporal profile of neutrals. Since, in this case, the ADE is close to the most intense absorption band of the spectrum (associated to an active excited state of the anion), the detachment threshold is obscured by the very intense signal of neutral fragments. The spectral location of the ADE is more clearly visible by plotting the difference yield of neutral particles (i.e. unfragmented - fragmented neutrals) as a function of the excitation energy, as shown in Fig. SI-2, with an acceptable signal-tonoise ratio. Using this procedure, a value of $280.0 \pm 0.5 \mathrm{~nm}(4.42 \pm 0.01 \mathrm{eV})$ was assigned to the ADE of the phenylphosphate anion.

\section{Calculations}

We have calculated adiabatic detachment energies, as well as dissociation energies, and vertical transitions to the anion excited states, to assist in the interpretation of the experimental results. In doing that, we have used different calculation methods.

ADE values were calculated using the Gaussian suite ${ }^{30}$, employing a (DFT/CAM-B3Lyp/aug-ccpVDZ) method to optimise the deprotonated anion and the dehydrogenated radical of the corresponding acids, i.e. phenylphosphonic, phenylsulfonic, p-toluenesulfonic, and phenylphosphoric acids. The results are presented Table 1. The calculations predict lower values than those obtained experimentally. There are two possible explanations for this: either the experiment cannot detect the energy threshold properly, because the signal is obscured by the presence of neutral fragments (originating from the excited anions), or the computation method is not adequate for these species, even though it is more accurate than the MP2 or CC2 methods which do not work for the radical ${ }^{31}$. We are confident that the experimental ADE values found for the aromatic anions of interest are correct, because they compare well with photoelectron spectroscopy studies made on phosphorus (or sulfur) inorganic monoanions, keeping the same oxidation state in each case (phosphorus $V$, as in phosphonate and phosphate, and sulfur VI, as in sulfonate). That is, the measured ADE values of aromatic phosphonate and phosphate are very close to that of dihydrogen phosphate $\left(\mathrm{H}_{2} \mathrm{PO}_{4}^{-}\right), 4.57$ $\mathrm{eV}^{9}$ and in the case of aromatic sulfonates the measured values are like that of hydrogen sulfate $\left(\mathrm{HSO}_{4}{ }^{-}\right), 4.75 \mathrm{eV}^{10}$

In the case of phenylphosphate, the $-\mathrm{OH}$ group can lie either in the phenyl plane or perpendicular to it, the former structure being more stable by $0.02 \mathrm{eV}$ at the (B3Lyp/aug-cc-pVDZ) level. Conversely, for phenylphosphonate, the "planar" configuration of the -OH group is not a stable structure, and the molecule adopts a "perpendicular" form upon optimization.

In the last column of Table 1, we list the calculated dipole moment values for the radicals of interest. Since all the values are well above $2.5 \mathrm{D},{ }^{19,20}$ all these structures can - in principle - form DBSs. 
Table 1. Adiabatic detachment energy (in eV) determined in the experiment ( $A D E_{\text {exp }}$ ) and calculated (ADE calc) at the (DFT/CAM-B3Lyp/aug-cc-pVDZ) level. In the last column are listed the dipole moments of the unfragmented radical, as calculated in the optimised geometry.

\begin{tabular}{|l|c|c|c|}
\hline Oxyanion & ADE $_{\text {exp }}$ & ADE $_{\text {calc }}$ (DFT/CAM-B3Lyp) & Radical dipole (D) \\
\hline phenylphosphonate & $4.60 \pm 0.05^{*}$ & 3.93 & 4.0 \\
\hline phenylphosphate & 4.42 & 4.05 & 8.8 \\
\hline phenylsulfonate & 4.89 & 4.12 & 3.9 \\
\hline p-toluenesulfonate & 4.74 & 4.42 & 6.3 \\
\hline
\end{tabular}

* For phenylphosphonate, $A D E_{\text {exp }}$ is obtained from the analysis of the DBS spectrum (see Fig. 5) whereas, for the rest of the anions, $A D E_{\exp }$ is obtained by the increase in the yield of the unfragmented radical remaining post electron PD.

The dissociation energies of the anions of interest have been calculated with Turbomole ${ }^{32}$ (DFT/B3Lyp/aug-cc-pVDZ), and the results are presented in Table 2. In the case of phosphorus oxyanions, the observed charged fragment is $\mathrm{PO}_{3}{ }^{-}$, which implies that fragmentation occurs after a reorganization of the ion. The stable benzene molecule is then released from phenylphosphonate, whereas phenol is formed in the case of phenylphosphate. These reactions are quite exoenergetic, reaching an excess energy of about $4 \mathrm{eV}$ upon excitation on the phenyl $\pi \pi^{*}$ transition (vide infra). On the other hand, fragmentation of phenylsulfonates does not require any atomic rearrangement, and it simply occurs via a C...S homolytic rupture. In this case, the released energy is smaller, estimated about $1 \mathrm{eV}$, upon excitation of the phenyl ring.

Table 2. Dissociation energies (eV) at the DFT/B3Lyp/aug-cc-pVDZ level

\begin{tabular}{|l|c|c|}
\hline & Dissociation channel & Dissociation energy \\
\hline phenylphosphonate & $\mathrm{C}_{6} \mathrm{H}_{5} \cdots \mathrm{PO}_{3} \mathrm{H}^{-}$ & 0.47 \\
\hline phenylphosphate & $\mathrm{C}_{6} \mathrm{H}_{5}-\mathrm{O} \cdots \mathrm{PO}_{3} \mathrm{H}^{-}$ & 1.08 \\
\hline phenylsulfonate & $\mathrm{C}_{6} \mathrm{H}_{5} \cdots \mathrm{SO}_{3}{ }^{-}$ & 2.81 \\
\hline p-toluenesulfonate & $\mathrm{CH}_{3}-\mathrm{C}_{6} \mathrm{H}_{4} \cdots \mathrm{SO}_{3}^{-}$ & 2.80 \\
\hline
\end{tabular}

Anion vertical excited states energies were calculated at the $A D C(2)$ or $C C 2$ levels, using the aug-cc-pVDZ basis set ${ }^{32}$. TD-DFT (B3Lyp or CAM-B3Lyp) gave values in quite strong disagreement with the experimental ones. In Table 3, we present only the CC2 values. $A D C(2)$ values are not included, because they are quite similar to the CC2 calculations, being on average $0.12 \mathrm{eV}$ lower. For the sulfonates, the excited states have been calculated with a Cs symmetry, and this allows us to readily distinguish the $\pi \pi^{*}$ states localized on the aromatic part ( $\mathrm{A}^{\prime}$ symmetry) from the charge transfer (CT) states in which the electron is transferred from the $-\mathrm{SO}_{3}{ }^{-}$group to some diffuse $\sigma^{*}$ orbital on the aromatic moiety. This is also reflected by the magnitude of the oscillator strengths, which are one or two orders of magnitude lower for the CT ( $A^{\prime \prime}$ symmetry) states. In the case of the phosphate anion, the absence of symmetry prohibits performing this simple analysis, but the oscillator strength values are still a good indicator of the properties of the excited states. For the phosphate, the lowest excited state is found to have CT character, together with a weak oscillator strength and, consequently, the first $\pi \pi^{*}$ state corresponds to the second excited state. 
Table 3. Excited state energies, calculated at the MP2 ground and CC2 excited state. The first $\pi \pi^{*}$ state is in each case indicated in bold type.

\begin{tabular}{|l|c|c|c|c|}
\hline \multirow{2}{*}{} & \multicolumn{2}{|c|}{ phenylphosphate } & \multicolumn{2}{c|}{ phenylphosphonate } \\
\cline { 2 - 5 } & energy (eV) & oscillator strength & energy (eV) & oscillator strength \\
\hline S1 & 4.29 & $3 \mathrm{E}-03$ & 4.53 & $7 \mathrm{E}-03$ \\
\hline S2 & 4.71 & $3 \mathrm{E}-02$ & 4.6 & $1 \mathrm{E}-02$ \\
\hline S3 & 4.73 & $3 \mathrm{E}-03$ & 4.82 & $6 \mathrm{E}-03$ \\
\hline S4 & 4.99 & $8 \mathrm{E}-03$ & 4.88 & $3 \mathrm{E}-03$ \\
\hline S5 & 5.13 & $1 \mathrm{E}-03$ & 4.93 & $5 \mathrm{E}-03$ \\
\hline S6 & 5.27 & $2 \mathrm{E}-04$ & 4.96 & $3 \mathrm{E}-03$ \\
\hline S7 & 5.4 & $9 \mathrm{E}-03$ & 4.99 & $3 \mathrm{E}-03$ \\
\hline
\end{tabular}

\begin{tabular}{|c|c|c|c|c|}
\hline \multirow{2}{*}{} & \multicolumn{2}{|c|}{ phenylsulfonate } & \multicolumn{2}{c|}{-toluenesulfonate } \\
\cline { 2 - 5 } & energy (eV) & oscillator strength & energy (eV) & oscillator strength \\
\hline $1 A^{\prime}$ & 4.58 & $3 \mathrm{E}-03$ & 4.51 & $1 \mathrm{E}-03$ \\
\hline $2 \mathrm{~A}^{\prime}$ & 4.9 & $8 \mathrm{E}-04$ & 4.79 & $5 \mathrm{E}-04$ \\
\hline $3 \mathrm{~A}^{\prime}$ & 4.94 & $1 \mathrm{E}-03$ & 4.84 & $9 \mathrm{E}-03$ \\
\hline $4 \mathrm{~A}^{\prime}$ & 5.07 & $1 \mathrm{E}-03$ & 5.03 & $1 \mathrm{E}-03$ \\
\hline $5 \mathrm{~A}^{\prime}$ & 5.33 & $2 \mathrm{E}-01$ & 5.04 & $6 \mathrm{E}-04$ \\
\hline $6 \mathrm{~A}^{\prime}$ & 5.41 & $2 \mathrm{E}-03$ & 5.12 & $1 \mathrm{E}-01$ \\
\hline $1 \mathrm{~A}^{\prime \prime}$ & 4.21 & $8 \mathrm{E}-06$ & 4.17 & $1 \mathrm{E}-08$ \\
\hline $2 \mathrm{~A}^{\prime \prime}$ & 4.51 & $3 \mathrm{E}-05$ & 4.45 & $3 \mathrm{E}-05$ \\
\hline $3 \mathrm{~A}^{\prime \prime}$ & 4.68 & $5 \mathrm{E}-05$ & 4.54 & $9 \mathrm{E}-05$ \\
\hline
\end{tabular}

\section{Discussion}

\subsection{Measurement of ADEs. Anionic excited state or DBS?}

Below the ADE threshold, any band appearing in the spectrum involves an excited state of the anion. Neutral detection is sensitive to such transitions because they mostly result in the fragmentation of the anion, producing a neutral and a charged particle. This process is most often due to internal conversion that leads to statistical fragmentation. The expected kinetic energy $\left(E_{\mathrm{kin}}\right)$ released in the fragmentation will be distributed according to the function $\exp \left(-a E_{\mathrm{kin}}\right)$, and this will be reflected in quite typical temporal profiles, such as those shown in Fig. 4 for phenylphosphate upon excitation on the first intense band. The excess of kinetic energy, estimated from the difference of excited state (Table 3 ) and dissociation energies (Table 2), adopt values as large as $4.1 \mathrm{eV}$ for phenylphosphate or $1.8 \mathrm{eV}$ for phenylsulfonate, causing a substantial broadening in the temporal 
profile of neutrals. This behaviour has been observed for all the studied anions (lowest energy band excitation), except in the case of phenylphosphonate.

Above the $A D E$, the excited states of the anion can autoionise faster than they fragment. Hence, for each excited state, there is a competition between producing the corresponding stable neutral radical (temporal profile as a narrow peak) or neutral fragments (broad temporal profile). This is the reason why the appearance of a narrow central contribution in the temporal profile of neutral particles can be used to determine the spectral position of the ADE. Clear ADE thresholds were observed for phenylsulfonate at $4.89 \mathrm{eV}(253.5 \mathrm{~nm})$ in Fig. SI-3, p-toluenesulfonate at $4.75 \mathrm{eV}$ $(261.5 \mathrm{~nm})$ in Fig. SI-4, and also for phenylphosphate at $4.42 \mathrm{eV}(280.5 \mathrm{~nm})$ in Fig. SI-2. A different approach is, however, employed to determine the ADE of phenylphosphonate at $4.60 \mathrm{~V}(269.5 \mathrm{~nm})$, as we will discuss later in section 5.3. Remarkably, these results are not so different from the ADE values of the simple inorganic dihydrogen phosphate $\mathrm{PO}_{4} \mathrm{H}_{2}{ }^{-}(4.57 \mathrm{eV})$ or hydrogen sulfate $\mathrm{HSO}_{3}{ }^{-}$ $(4.75 \mathrm{eV})$ anions, evidencing that the addition of such negatively charged substituents to an aromatic ring does not change drastically the ADE. This can be justified in terms of the negative charge being well localised on the oxyanion group, in accordance with the shape of the HOMO isosurface diagrams of phenylphosphonate, phenylsulfonate and p-toluenesulfonate (see Fig. SI-5). However, phenylphosphate does not behave in the same way, and the HOMO of this anion is more localised on the phenyl part.

In general, autoionisation is quite slow near the threshold, and the PF process can still occur above the ADE for about $0.5 \mathrm{eV}$. Beyond this limit, autoionisation generally becomes the fastest process, and the temporal profile of neutrals adopts the shape of a narrow peak. Within this transition, the profile adopts a complex form, with both broad and narrow contributions. The sequence starting in a broad peak, from which at a certain point a narrow peak emerges, until finally becoming a single narrow contribution, can be observed in Figs. 2-4 for the three aforementioned anions (except for phenylphosphonate, where a stable DBS rules the photophysics of this anion).

In the special case of the presence of a DBS, different behaviour is expected. Above the ADE, the loosely bound electron that characterises the DBS is easily autodetached, and a stable radical is left with almost no $E_{\text {kin }}$ release. Thus, the temporal profile of a radical originated in a DBS is expected to be a single narrow peak. This is exactly the case found for the phenylphosphonate anion (inset in Fig. 1). Even considering that a minor broad component could be present, we can be sure that this effect is not originating from any dissociation process involving the anion, because no bands are distinguished in the ion fragment spectrum (Fig. SI.1).

Another argument supporting the formation of a DBS by excitation of phenylphosphonate is the lack of a broad (dissociative) temporal profile, even at excitation energies as high as $230 \mathrm{~nm}$ (large $E_{\text {kin }}$ release), which confirms the high stability of the phenylphosphonate radical. For such a high photon energy, electron PD should likely be the most important relaxation channel, producing an unfragmented radical with, potentially, a lot of internal energy. Although photoelectron spectroscopy studies would be necessary to quantify this energy, we can anticipate that at the energies reached in our experiment the phenylphosphonate radical remains stable.

\subsection{Photofragmentation vs. photodetachment processes}

In this section, we will discuss how the experimental conditions affect the relative yield of the PF and PD processes. In particular, the prevalence of any of these channels varies when comparing our experiment to other studies on molecular anions performed at room temperature ${ }^{13,33-36}$, in which PF is usually a minor channel compared to PD. The first experimental condition to be analysed concerns the (vibrational) temperature of the ions. Indeed, PF efficiency is mostly controlled by this factor. The temperature effect on the PF yield is very easily studied on protonated aromatic species (in our case around $50 \mathrm{~K}^{37}$ ). When the molecules are cold, the observed spectrum is well resolved, and hence the vibrational states can be singled out for laser excitation, opening an efficient fragmentation channel. However, for hot molecules, the ground state ion population is spread over 
many rovibrational levels, decreasing PF efficiency. For protonated species, we have estimated that the PF yield (upon excitation of a specific absorption band) increases around two orders of magnitude when the trap is cooled down with a helium cryostat, in comparison with being at room temperature conditions. On the other hand, PD efficiency will not be influenced by the temperature, except very near the ADE, since in the process an electron is promoted to the continuum. Thus, the low fragmentation efficiency often measured in room temperature studies is more linked to the drawback of using a hot trap than to specific molecular properties. Finally, it is worth mentioning that even when dealing with cold molecular anions, it is possible to observe the absence (up to a detection limit below $1 \%$ ) of PF, as in the case of phenylphosphonate. The stability of this molecular anion upon excitation cannot be explained on the basis of a particularly rigid structure or because the excited states are inaccessible, since the dissociation energy calculated for this species is very low (Table 2) and the lowest lying excited states are not particularly high in energy (Table 3 ). It is thus the efficient autodetachment from phenylphosphonate's DBS that provides the relaxation mechanism avoiding fragmentation in this anion.

\subsection{Vibrational structures}

\section{Phenylsulfonate, $p$-toluenesulfonate and phenylphosphate}

The vibration bands found for these anions have been assigned to their respective excited states. The same spectral structure is found in both ion fragment and (profile edge) neutral detection schemes. The main vibrational signatures are shown in Fig. 5 and listed in Table 4 . It is found that the most intense bands -in particular, those corresponding to the sulfonates- are very similar to those found for the neutral benzene molecule. This is a good experimental indication that the excitation is localized on the aromatic part of the molecular anion. For phenylphosphate, however, the situation is less clear, and it is difficult to perform the assignation due to the presence of a nearby intense $S_{2}$ state. 


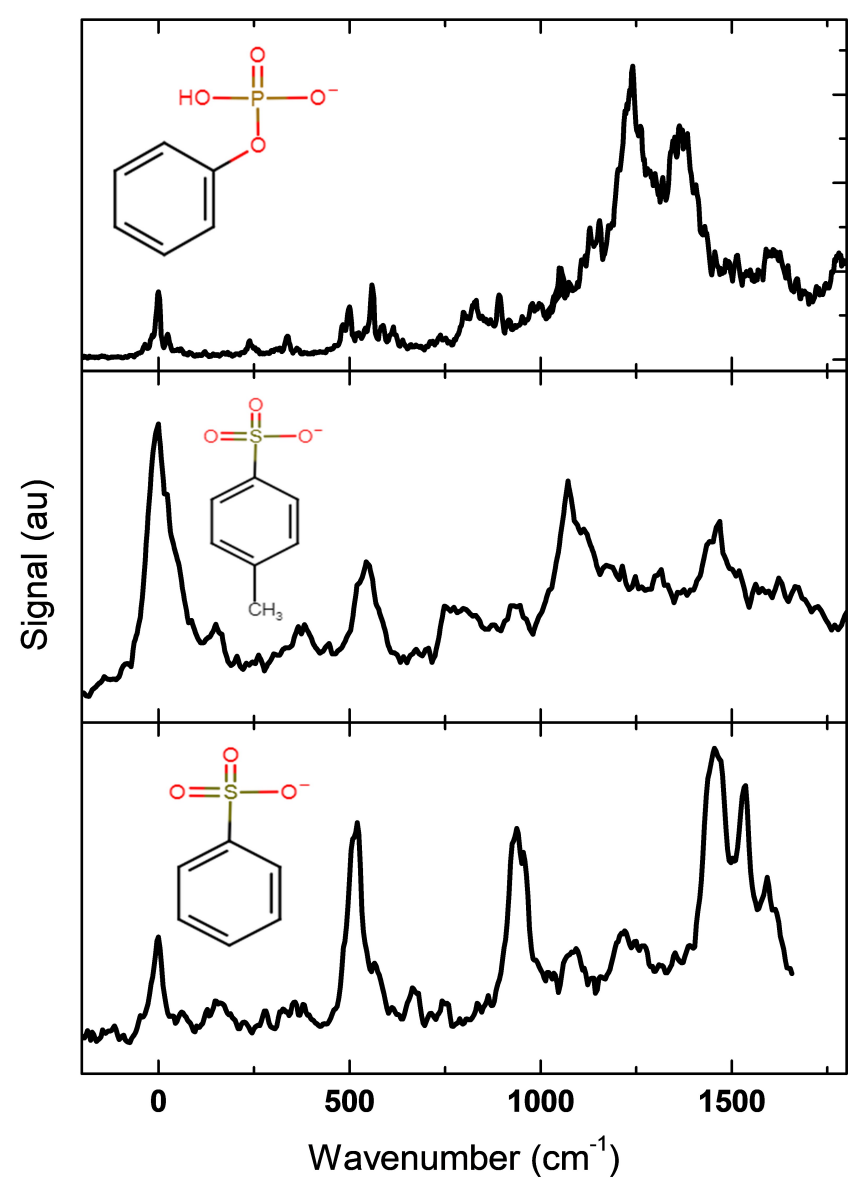

Figure 5. Onset of the first absorption band of phenylphosphate, p-toluenesulfonate, and phenylsulfonate, showing the vibrational structure of the corresponding excited states. Assigned $0-0$ bands at $34388 \mathrm{~cm}^{-1}$ $(290.80 \mathrm{~nm}, 4.26 \mathrm{eV}), 36947 \mathrm{~cm}^{-1}(270.66 \mathrm{~nm}, 4.58 \mathrm{eV})$ and $36258 \mathrm{~cm}^{-1}$ (275.80 nm, $\left.4.50 \mathrm{eV}\right)$ for phenylphosphate, phenylsulfonate and p-toluenesulfonate, respectively.

Table 4: Observed most intense vibrations in $\mathrm{cm}^{-1}$ compared to those of benzene $e^{38}$ in the 1 La state

\begin{tabular}{|c|c|c|c|c|}
\hline & phenylsulfonate & p-toluenesulfonate & phenylphosphate & benzene \\
\hline$v 6$ & 520 & 546 & 497 or 560 & 520 \\
\hline$v 1$ & 937 & 1074 & & 923 \\
\hline$v 14$ & 1460 & 1460 & & 1566 \\
\hline
\end{tabular}

\section{Pheny/phosphonate, as a special case}

Unlike the behaviour of the preceding molecular anions, the vibrational structure of phenylphosphonate, shown in Fig. 6, must be explained in terms of the formation of a DBS (as was already discussed in Section 5.1). A question arises as to whether we can use the vibrations of the DBS to make an estimation of the ADE value of phenylphosphonate. Considering that all the observed vibrations must in principle lie above the ADE, the lowest energy vibration can then be used as an upper limit value of the ADE. Since the first band clearly observed in the spectrum is at $37170 \mathrm{~cm}^{-1}$, we can attribute an energy value immediately below to the ADE of phenylphosphonate, proximate to $37100 \mathrm{~cm}^{-1}(4.60 \mathrm{eV})$. The $0-0$ band assigned to the DBS is very weak and located at $36804 \mathrm{~cm}^{-1}(4.56 \mathrm{eV})$. Above the $0-0$ band, there are vibrational modes at $356 \mathrm{~cm}^{-1}$, similar to that in 
the hydroxyphenoxide anion ${ }^{23}$, at $890 \mathrm{~cm}^{-1}$, and a progression at about $920 \mathrm{~cm}^{-1}$. Additionally, a very low energy progression at $70 \mathrm{~cm}^{-1}$ is evident, starting on an intense vibration at $37694 \mathrm{~cm}^{-1}$ (indicated by a star in the figure). Based on the vibrational spectrum calculated for the ground state of the anion, the $70 \mathrm{~cm}^{-1}$ vibration can be assigned to the pseudo rotation of the $-\mathrm{PO}_{3} \mathrm{H}^{-}$group, calculated at $50 \mathrm{~cm}^{-1}$.

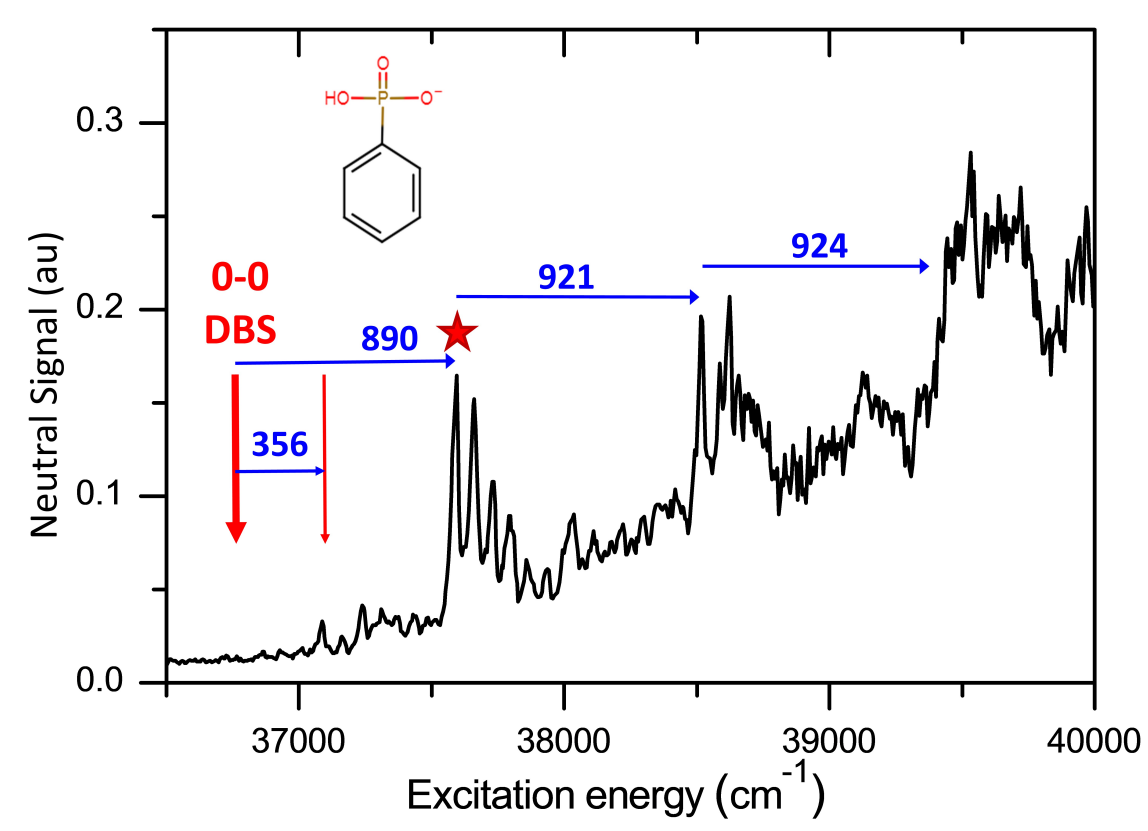

Figure 6. Onset of the first absorption band of phenylphosphonate, showing the vibrational structure of the DBS. As in Fig. 1, the first intense band at $37694 \mathrm{~cm}^{-1}(265.3 \mathrm{~nm})$ is marked by a star. The 0-0 band of the DBS is very weak and located at $36804 \mathrm{~cm}^{-1}$. Vibrational bands are assigned at $356 \mathrm{~cm}^{-1}$ and a progression at approximately $920 \mathrm{~cm}^{-1}$. The spectral position of the ADE can be estimated to lie immediately below the first observed vibrational band, around $37100 \mathrm{~cm}^{-1}$ (4.60 eV). 


\subsection{Anion excited states}

\section{Phenylphosphate}

The first two bands in Fig. 4 for this anion can definitively be assigned to anionic excited states, based on the broad temporal profiles of neutrals upon excitation on each one of them. The first one is weak, and it starts at $290.8 \mathrm{~nm}(4.26 \mathrm{eV})$. With the help of Table 3, we can assign this band to a CT state, which is consistent with the observation of having a low oscillator strength. The second band, starting at $280.68 \mathrm{~nm}(4.42 \mathrm{eV})$ can be assigned to the $\pi \pi^{*}$ state $\left(1 \mathrm{~A}^{\prime}\right)$, in accordance with the calculations. Finally, a third band structure with high intensity is exhibited in Fig. 4, starting at $240 \mathrm{~nm}$ $(5.16 \mathrm{eV})$, which probably may be attributed to the $5 \mathrm{~A}^{\prime}$ excited state according to the calculated strong oscillator strength.

\section{Phenylsulfonate and p-toluenesulfonate}

The spectra of these two ions are very similar to each other: only one vibrational band structure starting at $270.66 \mathrm{~nm}(4.58 \mathrm{eV})$ for phenylsulfonate and at $275.8 \mathrm{~nm}(4.50 \mathrm{eV})$ for toluenesulfonate is clearly observed. At higher energy, very broad bands are seen at $255 \mathrm{~nm}(4.86$ $\mathrm{eV})$ and at $235 \mathrm{~nm}(5.28 \mathrm{eV})$ for phenylsulfonate and at $250 \mathrm{~nm}(4.96 \mathrm{eV})$ and $235 \mathrm{~nm}(5.28 \mathrm{eV})$ for toluenesulfonate. They can be tentatively assigned to the $1 A^{\prime}, 3 A^{\prime} 4 A^{\prime}$ (quasi degenerated) and $5 A^{\prime}$ excited states for the first anion, and to the $1 A^{\prime}, 4 A^{\prime}$ and $6 A^{\prime}$ excited states for the second. All these excited states are localised on the aromatic part.

\subsection{Effect of the functional group on the $\pi \pi^{*}$ transition energy}

Is the energy of the $\pi \pi^{*}$ state of the anions sensitive to the presence of a negatively charged phosphorus or sulfur functional group? For the moment, we have a very limited set of data to answer this question ( $0-0$ bands at $4.42,4.58$ and $4.50 \mathrm{eV}$ for phenylphosphate, phenylsulfonate and $\mathrm{p}$ toluenesulfonate, respectively), but nonetheless one can see that the energy change of the $\pi \pi^{*}$ transition upon the addition of different oxyanions is not large (Fig. 7). Moreover, we will show now that the very presence of an oxyanion on the aromatic structure only has a minor effect on the transition energy. For doing that, one can compare, for example, the energy of the $\pi \pi^{*}$ state of the phenylphosphate anion to that of neutral phenol (which shares a Ph-O- structure, and has a transition energy of $4.5 \mathrm{eV}$ ) and, in the case of the aromatic sulfonates, to the $\pi \pi^{*}$ state of the related molecules benzene $(4.72 \mathrm{eV})$ or toluene $(4.66 \mathrm{eV})$. In Fig. 7 , it is observed that substitution with phosphorus and sulfur oxyanions only produces a small red shift in the $\pi \pi^{*}$ transition (of about $0.10 \mathrm{eV}$ for phenylphosphate and $0.15 \mathrm{eV}$ for the aromatic sulfonates). These results are in line with the observed red shift on the $\pi \pi^{*}$ transition of molecules with different dipolar substituents ${ }^{39}$, but they are in contrast with studies performed on the phosphorylation of peptides containing tyrosine, in which a blue shift of $\sim 0.15 \pm 0.05 \mathrm{eV}$ is induced in the tyrosine band (phosphotyrosine $4.6 \mathrm{eV}$ ). ${ }^{7}$ 


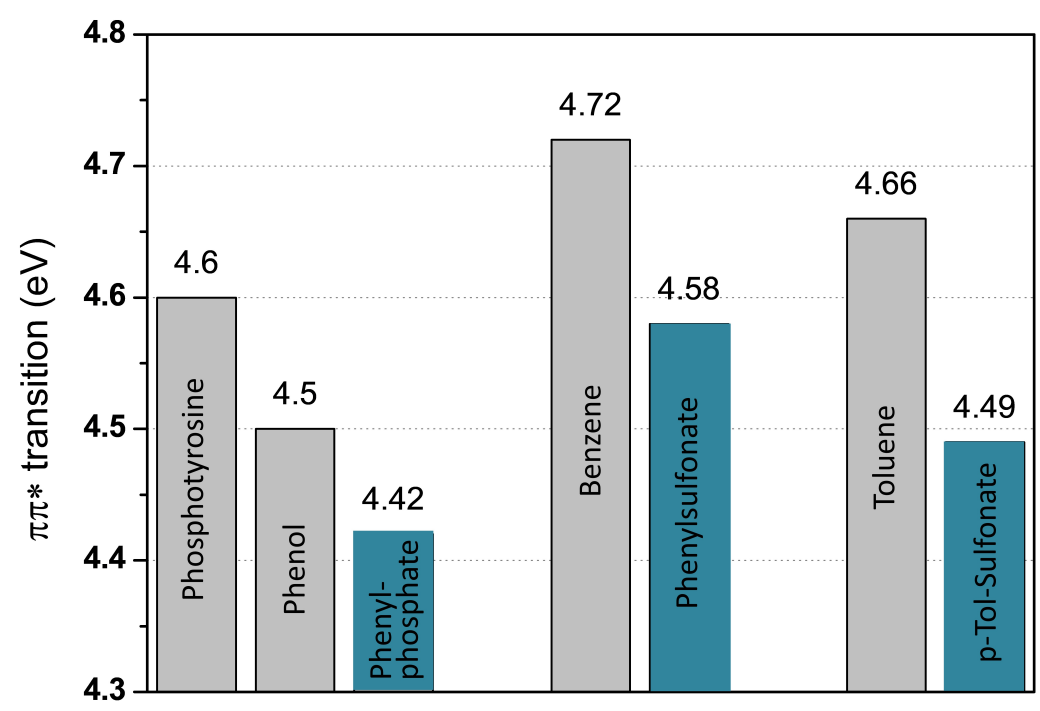

Figure 7. Comparison of $\pi \pi^{*}$ transition energies among negatively charged substituted aromatic ions (blue) and the corresponding neutral aromatic chromophores (grey). For phosphotyrosine the value is taken from Kopisov et $a l^{7}$

\section{Conclusions}

Phenylphosphate, phenylsulfonate and p-toluenesulfonate exhibit the expected photophysics behaviour for aromatic anions: at low energy, excitation into anionic excited states leads to fragmentations. The observed states mainly originate in $\pi \pi \rightarrow \pi \pi^{*}$ transitions on the aromatic moieties, except for phenylphosphate, in which a weak CT state is observed at somewhat lower energy. As soon as the ADE is reached, the anion may lose an electron to form a stable radical but it also may fragment from the excited states that are available. At higher energy, the latter process is no longer competitive with electron detachment and photofragmentation no longer occurs. Even though phenylphosphate and phenylphosphonate anions share the same $-\mathrm{PO}_{3}{ }^{-}$moiety and they are both aromatic phosphorus oxyanions with the same oxidation state, the latter does not exhibit transitions to anionic excited states. Instead, phenylphosphonate evidences a band structure upon excitation that can be explained in terms of the formation of a DBS. However, since the calculations support the existence of anionic excited states within this spectral range, the absence of such transitions may be due to their very weak Frack Condon factors. This implies that the excited states of phenylphosphonate may have a very different equilibrium geometry, probably due to a $90^{\circ}$ rotation of the $-\mathrm{PO}_{3}{ }^{-}$group along the $\mathrm{C}-\mathrm{P}$ axis.

\section{Acknowledgments}

This work has been conducted within the International Associated Laboratory LEMIR (CNRS/CONICET) and was supported by CONICET, FONCYT, SECYT-UNC, CNRS (INSIS), and the ANR Research Grant (ANR2010BLANC040501-ESPEM, ANR17CE05000502-Wsplit and ANR-16-CE29-0017). The authors acknowledge the use of the computing facility cluster Meso-LUM of the LUMAT federation (LUMAT FR 2764). 


\section{References}

1 G. A. Pino, R. A. Jara-Toro, J. P. Aranguren-Abrate, C. Dedonder-Lardeux and C. Jouvet, Dissociative photodetachment vs. photodissociation of aromatic carboxylates: the benzoate and naphthoate anions, Phys. Chem. Chem. Phys., 2019, 21, 1797-1804.

2 J. A. Noble, J. P. Aranguren-Abate, C. Dedonder, C. Jouvet and G. A. Pino, Photodetachment of deprotonated aromatic amino acids: Stability of the dehydrogenated radical depends on the deprotonation site, Phys. Chem. Chem. Phys., 2019, 21, 23346-23354.

3 J. C. Rienstra-Kiracofe, G. S. Tschumper, H. F. Schaefer, S. Nandi and G. B. Ellison, Atomic and molecular electron affinities: Photoelectron experiments and theoretical computations, Chem. Rev., 2002, 102, 231-282.

4 L. Goodwin, J. R. Startin, D. M. Goodall and B. J. Keely, Negative ion electrospray mass spectrometry of aminomethylphosphonic acid and glyphosate: Elucidation of fragmentation mechanisms by multistage mass spectrometry incorporating in-source deuterium labelling, Rapid Commun. Mass Spectrom., 2004, 18, 37-43.

5 R. W. Binkley, T. W. Flechtner, M. J. S. Tevesz, W. Winnik and B. Zhong, Rearrangement of Aromatic Sulfonate Anions in the Gas Phase, Org. mass Spectrom., 1993, 28, 769-772.

6 J. D. Smith, R. A. J. O'Hair and T. D. Williams, Gas phase chemistry of sulfonate anions: Basicities and fragmentation reactions, Phosphorus, Sulfur Silicon Relat. Elem., 1996, 1, 49-59.

7 V. Kopysov, N. S. Nagornova and O. V. Boyarkin, Identification of tyrosine-phosphorylated peptides using cold ion spectroscopy, J. Am. Chem. Soc., 2014, 136, 9288-9291.

8 J. H. Kwon, M. J. Lee, G. Song, K. Tsuruta, S. Ishiuchi, M. Fujii and H. Kang, Cryogenic lon Spectroscopy of a Singly Protonated Peptide DYYVVR: Locating Phosphorylation Sites of a Kinase Domain, J. Phys. Chem. Lett., 2020, 11, 7103-7108.

9 X. Bin Wang, E. R. Vorpagel, X. Yang and L. S. Wang, Experimental and theoretical investigations of the stability, energetics, and structures of H2PO4-, H2P2O72-, and H3P3O102- in the gas phase, J. Phys. Chem. A, 2001, 105, 10468-10474.

10 X. Bin Wang, J. B. Nicholas and L. S. Wang, Photoelectron spectroscopy and theoretical calculations of so4- and HSO4-: Confirmation of high electron affinities of so4 and HSO4, J. Phys. Chem. A, 2000, 104, 504-508.

11 V. M. Rivilla, M. N. Drozdovskaya, K. Altwegg, P. Caselli, M. T. Beltrán, F. Fontani, F. F. S. Van Der Tak, R. Cesaroni, A. Vasyunin, M. Rubin, F. Lique, S. Marinakis, L. Testi, H. Balsiger, J. J. Berthelier, J. De Keyser, B. Fiethe, S. A. Fuselier, S. Gasc, T. I. Gombosi, T. Sémon and C. Y. Tzou, ALMA and ROSINA detections of phosphorus-bearing molecules: The interstellar thread between star-forming regions and comets, Mon. Not. R. Astron. Soc., 2020, 492, 1180-1198.

12 A. M. Turner, A. Bergantini, M. J. Abplanalp, C. Zhu, S. Góbi, B. J. Sun, K. H. Chao, A. H. H. Chang, C. Meinert and R. I. Kaiser, An interstellar synthesis of phosphorus oxoacids, Nat. Commun., 2018, 9, 1-9.

13 L. Giacomozzi, C. Kjær, J. Langeland Knudsen, L. H. Andersen, S. Brøndsted Nielsen and M. H. Stockett, Absorption and luminescence spectroscopy of mass-selected flavin adenine dinucleotide mono-anions, J. Chem. Phys., 2018, 148, 214309.

14 J. Schiedt and R. Weinkauf, Resonant photodetachment via shape and Feshbach resonances: P-benzoquinone anions as a model system, J. Chem. Phys., 1999, 110, 304-314.

15 R. E. Continetti and H. Guo, Dynamics of transient species via anion photodetachment, Chem. Soc. Rev., 2017, 46, 7650-7667.

16 Z. Lu and R. E. Continetti, Dynamics of the acetyloxyl radical studied by dissociative photodetachment of the acetate anion, J. Phys. Chem. A, 2004, 108, 9962-9969.

17 R. D. Mead, K. R. Lykke, W. C. Lineberger, J. Marks and J. I. Brauman, Spectroscopy and dynamics of the dipole-bound state of acetaldehyde enolate, J. Chem. Phys., 1984, 81, 48834892.

18 J. Marks, J. I. Brauman, R. D. Mead, K. R. Lykke and W. C. Lineberger, Spectroscopy and dynamics of the dipole-supported state of acetyl fluoride enolate anion, J. Chem. Phys., 1988, 88, 6785-6792. 
C. Desfrançois, H. Abdoul-Carime, N. Khelifa and J. P. Schermann, From $1 / \mathrm{r}$ to $1 / \mathrm{r} 2$ potentials: Electron exchange between Rydberg atoms and polar molecules, Phys. Rev. Lett., 1994, 73, 2436-2439.

20 C. Desfrançois, H. Abdoul-Carime and J.-P. Schermann, Ground state dipole bound anions, Int. J. Mod. Physic B, 1996, 10, 1339-1395.

21 H.-T. Liu, C.-G. Ning, D.-L. Huang, P. D. Dau and L.-S. Wang, Observation of Mode-Specific Vibrational Autodetachment from Dipole-Bound States of Cold Anions, Angew. Chemie, 2013, 125, 9146-9149.

22 H.-T. T. Liu, C.-G. G. Ning, D.-L. L. Huang and L.-S. S. Wang, Vibrational spectroscopy of the dehydrogenated uracil radical by autodetachment of dipole-bound excited states of cold anions, Angew. Chemie - Int. Ed., 2014, 53, 2464-2468.

23 D. L. Huang, H. T. Liu, C. G. Ning and L. S. Wang, Conformation-selective resonant photoelectron spectroscopy via dipole-bound states of cold anions, J. Phys. Chem. Lett., 2015, 6, 2153-2157.

24 I. Alata, J. Bert, M. Broquier, C. Dedonder, G. Feraud, G. Grégoire, S. Soorkia, E. Marceca and C. Jouvet, Electronic spectra of the protonated indole chromophore in the gas phase., J. Phys. Chem. A, 2013, 117, 4420-7.

25 G. Féraud, C. Dedonder, C. Jouvet, Y. Inokuchi, T. Haino, R. Sekiya and T. Ebata, Development of Ultraviolet - Ultraviolet Hole-Burning Spectroscopy for Cold Gas-Phase Ions, J. Phys. Chem. Lett., 2014, 5, 1236-1240.

26 M. Berdakin, G. Féraud, C. Dedonder-Lardeux, C. Jouvet and G. A. Pino, Excited States of Protonated DNA/RNA Bases, Phys. Chem. Chem. Phys., 2014, 16, 10643-10650.

27 W. C. Wiley and I. . McLaren, Time-of-Flight Mass Spectrometer with Improved Resolution, Rev. Sci. Instrum., 1955, 26, 1150.

28 M. Barat, J. C. Brenot, J. A. Fayeton and Y. J. Picard, Absolute detection efficiency of a microchannel plate detector for neutral atoms, Rev. Sci. Instrum., 2000, 71, 2050.

29 F. B. Jariwala, R. E. Wood, U. Nishshanka and A. B. Attygalle, Formation of the bisulfite anion (HSO3-, $\mathrm{m} / \mathrm{z}$ 81) upon collision-induced dissociation of anions derived from organic sulfonic acids, J. Mass Spectrom., 2012, 47, 529-538.

30 M. J. Frisch, G. W. Trucks, H. B. Schlegel, G. E. Scuseria, M. A. Robb, J. R. Cheeseman, V. G. Zakrzewski, J. A. Montgomery, R. E. Stratmann, J. C. Burant, S. Dapprich, J. M. Millam, A. D. Daniels, K. N. Kudin, M. C. Strain, O. Farkas, J. Tomasi, V. Barone, M. Cossi, R. Cammi, B. Mennucci, C. Pomelli, C. Adamo, S. Clifford, J. Ochterski, G. A. . Petersson, P. Y. Ayala, Q. Cui, K. Morokuma, D. K. Malick, A. D. . Rabuck, K. Raghavachari, J. B. Foresman, J. Cioslowski, J. V Ortiz, B. B. Stefanov, G. Liu, A. Liashenko, P. Piskorz, I. Komaromi, R. Gomperts, R. L. Martin, D. J. Fox, T. Keith, M. A. Al-Laham, C. Y. Peng, A. Nanayakkara, C. Gonzalez, M. Challacombe, P. M. W. Gill, B. G. Johnson, W. Chen, M. W. Wong, J. L. Andres, M. Head-Gordon, E. S. Replogle, J. A. Pople, M. W. Schmidt, E. K. K. Baldridge, J. A. Boatz, S. T. Elbert, M. S. Gordon, J. H. Jensen, S. Koseki, N. Matsunaga, K. A. Nguyen, S. J. Su, T. L. Windus and M. DuPuis, Gaussian 09; 2019, Gaussian, Inc. Pittsburgh, PA.

31 R. Send, M. Kühn and F. Furche, Assessing excited state methods by adiabatic excitation energies, J. Chem. Theory Comput., 2011, 7, 2376-2386.

32 TURBOMOLE V6.6, a development of University of Karlsruhe and Forschungszentrum Karlsruhe $\mathrm{GmbH}$, 1989-2007, TURBOMOLE GmbH, since 2007; available from http://www.turbomole.com.

33 L. Lammich, M. A. Petersen, M. B. Nielsen and L. H. Andersen, The gas-phase absorption spectrum of a neutral GFP model chromophore., Biophys. J., 2007, 92, 201-7.

34 R. Cercola, E. Matthews and C. E. H. Dessent, Photoexcitation of Adenosine 5'-Triphosphate Anions in Vacuo: Probing the Influence of Charge State on the UV Photophysics of Adenine, $J$. Phys. Chem. B, 2017, 121, 5553-5561.

35 C. S. Anstöter and J. R. R. Verlet, Gas-Phase Synthesis and Characterization of the Methyl-2,2dicyanoacetate Anion Using Photoelectron Imaging and Dipole-Bound State Autodetachment, J. Phys. Chem. Lett., 2020, 11, 6456-6462. 
36 S. B. Nielsen, A. Lapierre, J. U. Andersen, U. V Pedersen, S. Tomita and L. H. Andersen, Absorption spectrum of the green fluorescent protein chromophore anion in vacuo, Phys. Rev. Lett., 2001, 87, 228102.

37 J. A. Noble, C. Dedonder and C. Jouvet, the Electronic Spectra of Protonated PANH Molecules, Astron. Astrophys., 2015, A79, 577.

38 K. Ohon, A study of Excited states molecular vibrations of aromatic hydrocarbons, Chem.Phys. lett, 1979, 64, 560.

39 J. Donon, S. Habka, V. Vaquero-Vara, V. Brenner, M. Mons and E. Gloaguen, Electronic Stark Effect in Isolated Ion Pairs, J. Phys. Chem. Lett., 2019, 10, 7458-7462. 


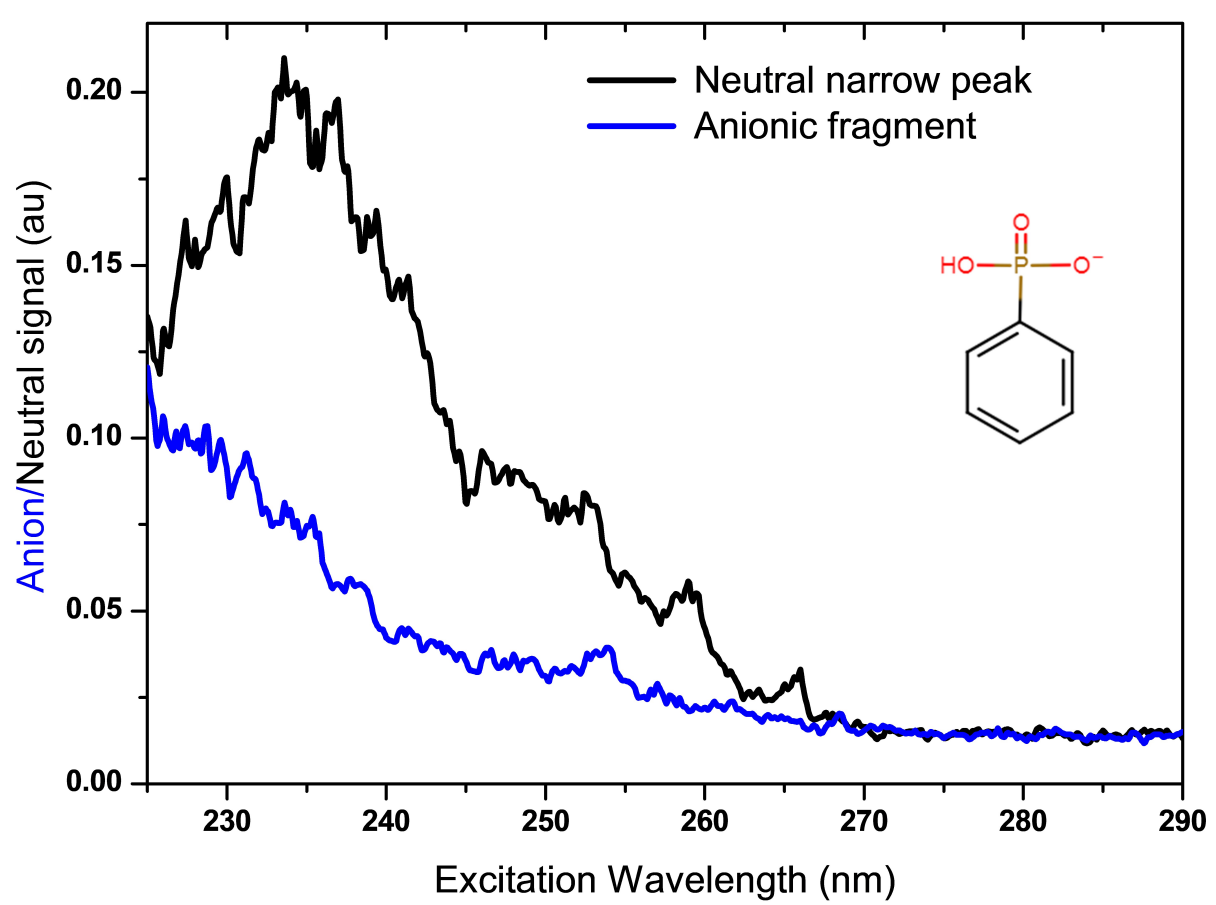

Figure SI-1. Ion yield (blue) and neutral particles yield (black) spectra of phenylphosphonate, as a measure of the extent of PF and PD channels, respectively. No bands are distinguishable in the ion yield spectrum, because the absorption does not involve any excited state of the anion (NB the resolution here is lower than in Fig. 1).

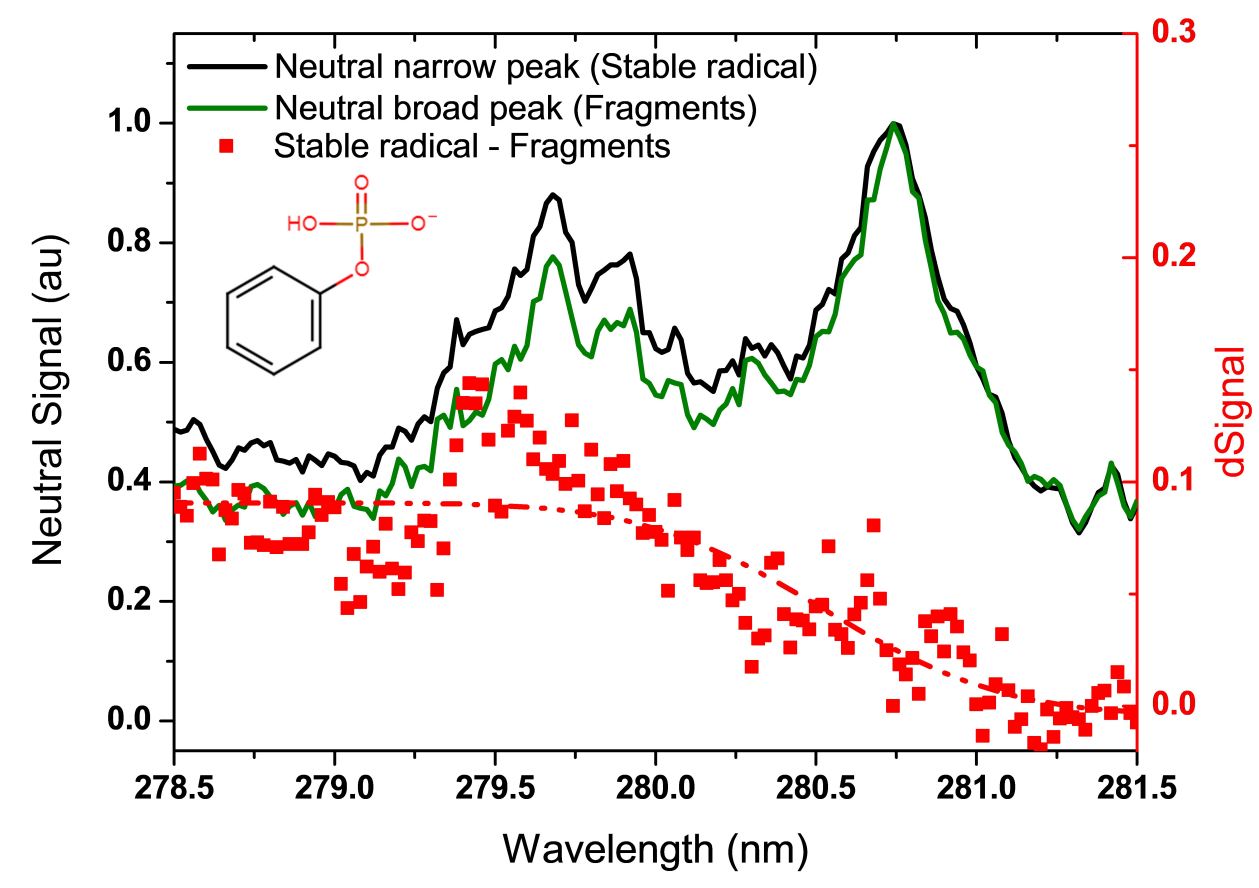

Figure SI-2. Phenylphosphate unfragmented precursor (black) and fragmented (green) neutrals as a function of the excitation wavelength. Signals are, respectively, measures of PD and PF channels. Differential yield between precursor and fragmented neutrals (red points, ordinate scale on the right); the increase of this quantity occurring between 280 and $281 \mathrm{~nm}$ delimits the value of the ADE of phenylphosphate. Notice that the differential yield is around ten times smaller than the photofragment signal. 


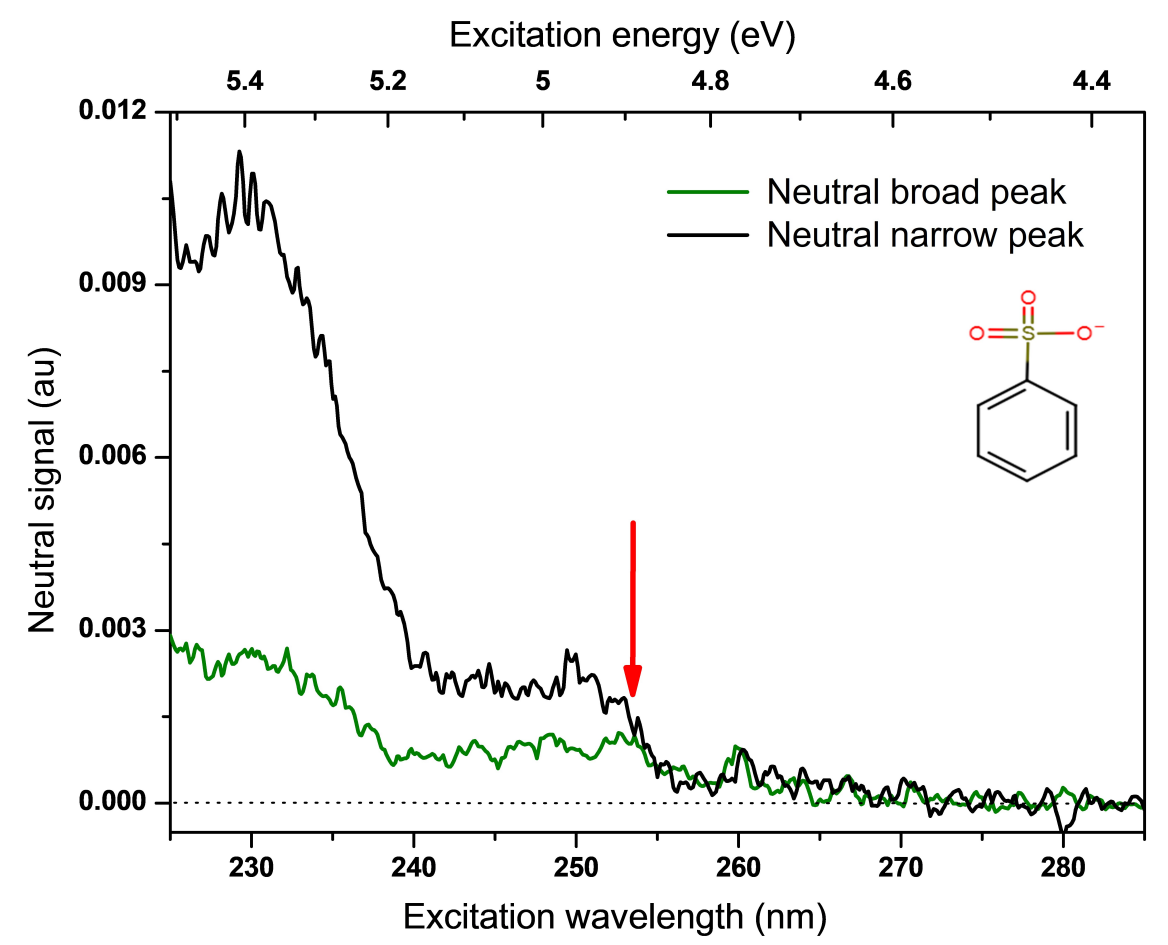

Figure SI-3. Determination of the spectral position of the ADE threshold for phenylsulphonate. The arrow indicates the appearance of a narrow central contribution in the temporal profile of neutral particles, corresponding to an $A D E=4.89 \mathrm{eV}(253.5 \mathrm{~nm})$.

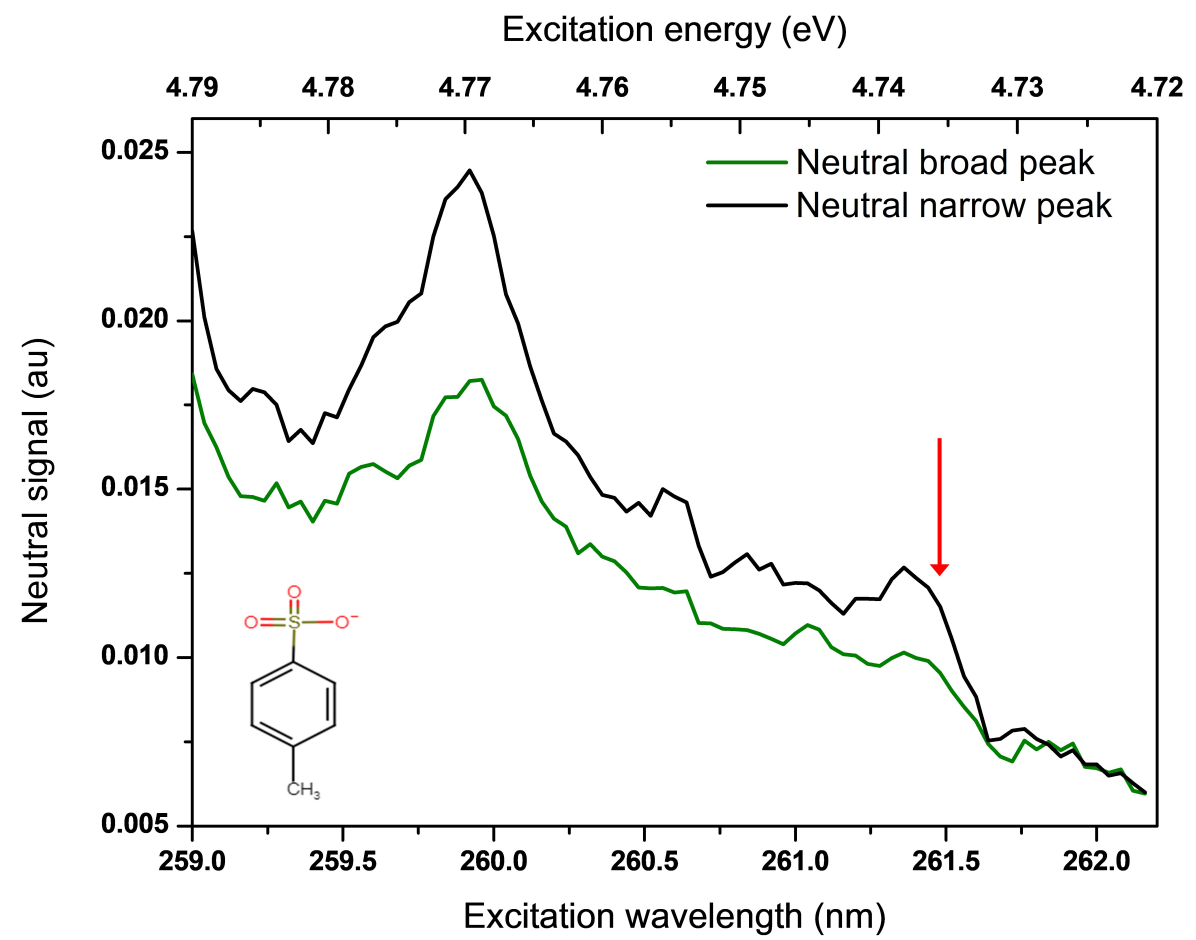

Figure SI-4. Determination of the spectral position of the ADE threshold for p-toluenesulfonate. The arrow indicates the appearance of a narrow central contribution in the temporal profile of neutral particles, corresponding to an $A D E=4.75 \mathrm{eV}(261.5 \mathrm{~nm})$. 

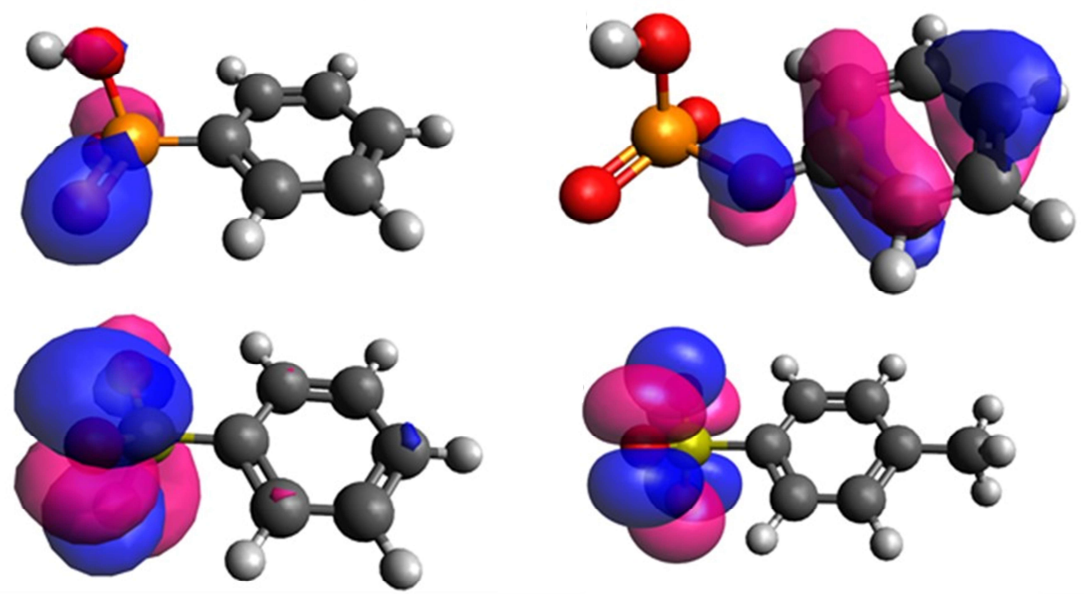

Figure SI-5. HOMOs of phenylphosphonate (upper left), phenylphosphate (upper right), phenylsulfonate (lower left) and p-toluenesulfonate (lower right). 